\title{
Stellar population properties for a sample of hard X-ray AGNs ${ }^{\star}$
}

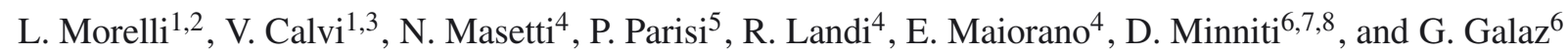 \\ 1 Dipartimento di Fisica e Astronomia “G. Galilei”, Università di Padova, vicolo dell'Osservatorio 3, 35122 Padova, Italy \\ e-mail: lorenzo.morelli@unipd.it \\ 2 INAF - Osservatorio Astronomico di Padova, vicolo dell'Osservatorio 5, 35122 Padova, Italy \\ 3 Space Telescope Science Institute, 3700 San Martin Drive, Baltimore, MD 21218, USA \\ ${ }^{4}$ INAF - Istituto di Astrofisica Spaziale e Fisica Cosmica di Bologna, via Gobetti 101, 40129 Bologna, Italy \\ 5 INAF - Istituto di Astrofisica e Planetologia Spaziali, via del Fosso del Cavaliere 100, 00133 Roma, Italy \\ ${ }^{6}$ Departamento de Astronomía y Astrofísica, Pontificia Universidad Católica de Chile, Casilla 306, Santiago 22, Chile \\ 7 Vatican Observatory, V00120 Vatican City State, Italy \\ 8 Departamento de Ciencias Fisicas, Universidad Andres Bello, Santiago, Chile
}

Received 18 April 2013 / Accepted 7 June 2013

\section{ABSTRACT}

\begin{abstract}
Aims. The aim of this paper is to study the stellar population of galaxies hosting an active galactic nucleus (AGN). We studied a subsample of hard X-ray emitting AGNs from the INTEGRAL and Swift catalogs, which were previously identified and characterized through optical spectroscopy. Our analysis provides complementary information, namely age and metallicity, which is necessary to complete the panoramic view of these interesting objects.

Methods. We selected hard X-ray emitting objects, identified as AGNs, by checking their optical spectra in search of absorption lines suitable for the stellar population analysis. We obtained a final sample consisting of 20 objects with a redshift lower than 0.3 . We used the full-spectrum fitting method; particularly, we use penalized pixel method and apply the PPXF code. After masking all the regions affected by emission lines, we fitted the spectra with the MILES single stellar population templates, and we derived mass-weighted ages and metallicities.

Results. Most of the objects in our sample show an old stellar population; however, three of them are characterized by a bimodal distribution with a non-negligible contribution from young stars. The values of the mass-weighted metallicity span a wide range with most of them slightly above the solar value. No relations between the stellar population properties and the morphological ones have been found.
\end{abstract}

Key words. galaxies: active - galaxies: evolution - galaxies: Seyfert - galaxies: stellar content - techniques: spectroscopic

\section{Introduction}

The massive work carried out by Masetti et al. (2004, 2006a,b,c,d, 2008, 2009, 2010, 2012), Landi et al. (2007), Parisi et al. (2009, 2012), and Maiorano et al. (2011) hereafter, Papers I-XIII supplied the astronomical community with a cat$\operatorname{alog}^{1}$ of hard X-ray sources for which the identification and the main physical parameters, computed using the multiwavelength information available in the literature, are provided. In total, more than 250 objects in the 20-200 keV range observed during the INTEGRAL (Winkler et al. 2003) and Swift (Gehrels et al. 2004) missions were studied and classified with $\sim 200$ of them using the IBIS instrument (Ubertini et al. 2003) and with $\sim 60$ using the BAT instrument (Barthelmy 2004). In particular, an optical follow-up to unveil the nature of most of these objects was mandatory, since only optical spectra permit an accurate source classification and provide fundamental parameters. In Papers I-XIII, we measured the flux of the most important emission lines existing in the optical part of the spectrum with the main aims of identifying the object and of investigating the properties of the host galaxies. For example, we measured properties, such as the Compton nature of these objects and an estimate of the mass of the central black hole in broad emission

\footnotetext{
* Table 1 and Figs. 3-5 are available in electronic form at http://www . aanda.org

1 The up-to-date version of this catalog is available on http://www.iasfbo.inaf.it/ masetti/IGR/main.html
}

line AGNs. Nevertheless, information regarding the stellar population of the galaxy hosting the AGNs is missing in the catalog. Therefore, stellar populations are new, important, and complementary pieces of information, which should be included in the catalog and which shed light on the properties of the AGNs and their host galaxies.

In the past decade, several authors focused their attention on the relation between the nuclear activity and the star formation rate (Ivanov et al. 2000; González Delgado et al. 2001; Joguet et al. 2001; Ho et al. 2003; La Mura et al. 2009; Draper \& Ballantyne 2011; Cracco et al. 2011; Vaona et al. 2012). This relation can give important clues about the fate of the gas that fuels the central black hole (Hopkins \& Hernquist 2006) and its influence on the central part of the host galaxy (Sarzi et al. 2005).

According to simulations (Di Matteo et al. 2005; Springel et al. 2005; Fontanot et al. 2011), a merging episode involving galaxies rich in gas induces radial gas inflows, which feed the black hole, and consequently, enhance the central star formation. Then, the AGN feedback wipes out basically all the remaining gas and dust (Hopkins \& Hernquist 2006; Rigopoulou et al. 2009), halting the star formation. However, the proposed scenarios in the past years became even more complicated. The model by Novak et al. (2011) showed that the AGN is a cyclic process. To account for the observational properties of this class of objects, it is necessary to consider different processes acting on different scales, such as: mechanic feedback that is a few pc from the nucleus, radiative feedback and consequent cooling flow of 
gas on a scale of few kpc, and SN winds, which are relevant on scales of tens of kpc (Ciotti et al. 2010).

Some observational works (Tremonti et al. 2007; Feruglio et al. 2010; McKernan et al. 2010) stated that AGNs could have played a relevant role in halting the star formation in massive host galaxies. Schawinski et al. (2007) suggested that the feedback could stop any star formation activity in the nucleus of the hosting galaxy and even beyond if the accretion of material onto the central black hole is powerful enough. As a consequence of this process, the stellar populations in the AGN host galaxies are old, and as suggested by Schawinski et al. (2007) and Faber et al. (2007), this causes the host galaxies to move from the blue cloud to the red sequence of the galaxy optical color diagram. Moreover, Bluck et al. (2011) computed the average energy output per galaxy due to AGN showing that this is at least 35 times larger than the binding energy of a typical massive galaxy.

Since an invaluable piece of information to understand the processes of formation and evolution of galaxies is imprinted in their stellar populations, some studies in recent years aimed to investigate the properties of the stellar populations of the host galaxy have been conducted. By studying the stellar populations in the nuclear region and their radial profile of a sample of AGNs that are mostly Seyfert 2 (hereafter Sy2), it was found that about one third of the galaxies in the sample shows an old bulge-like stellar population in the center (Ho et al. 2003; Cid Fernandes et al. 2004; Chen et al. 2009) and that the number of objects with an old stellar population increases to about two-thirds when the outer regions of low luminosity AGN hosts are investigated (Cid Fernandes et al. 2004).

In a recent paper, La Mura et al. (2012) investigated the connection between stellar population and mass in a large sample of type 1 and 2 AGNs. They found that the mass of the stellar component is a key ingredient to study the star formation history (Mannucci et al. 2010) of galaxies and taking this into account, they suggested an evolutionary sequence moving from starburst galaxies to AGNs (Davies et al. 2007; Schawinski et al. 2007).

Essentially, the observations show a variety of results even when they are restricted to the analysis of only Sy2 galaxies for which broad lines are weak or absent (Lawrence et al. 1987). This could be due to the difficulties in choosing homogeneous samples of objects and to an intrinsic complexity in interpreting the results, especially considering that the AGN theoretical model is still debated.

In this scenario, our results will be useful for understanding the properties of AGN hosts and the consequences of the AGN feedback on them, as well as to help in testing the predictions of theoretical models. The aim of this paper is, therefore, to present the stellar populations properties for a sample of AGN hosting galaxies, which were identified as the optical counterparts of hard X-ray emitting sources in Papers I-XIII.

In Sect. 2, we described the sample selection and the characteristics of the data. In Sect. 3, we illustrated the tools we used and the procedure we followed to obtain the final results. In Sect. 4, we presented and discussed our results concerning the stellar populations, in particular for the age and the metallicity derived from the stellar population fitting procedure. Finally, we summarized results and conclusions in Sect. 5.

\section{Sample selection and data properties}

\subsection{The sample}

The aim of this paper is to complete the catalog of optical counterparts of hard X-ray sources detected in the massive survey performed by our collaboration (Papers I-XIII), for which we

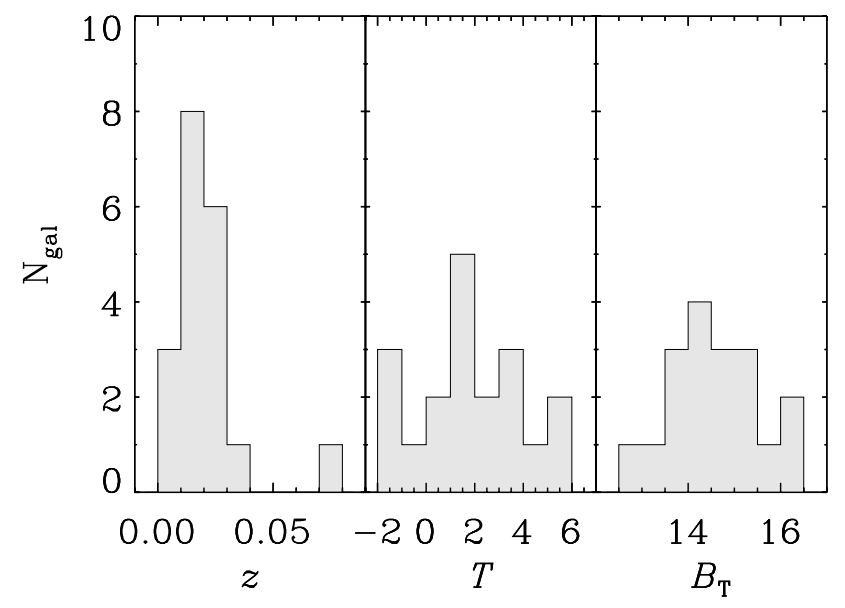

Fig. 1. Histograms showing the distribution of the main properties of the galaxies in our sample. From left to right, we show redshift $z$, morphological type $T$, and total magnitude in the $B$-band $B_{T}$.

have already detected and measured the emission line features with the study of the stellar population properties of the hosting galaxy. Consequently, complete information in the X-ray and optical range of the spectrum will be available for a subsample of objects in the catalog. To choose the galaxy sample, we started selecting all those objects identified as AGNs in Papers I-XIII. In detail, these hard X-ray sources were selected among those with an unidentified nature that belonged to the INTEGRAL and Swift surveys (e.g., Bird et al. 2010; Cusumano et al. 2010). The selection method consisted in choosing sources containing a single soft X-ray object within their arcmin-sized hard X-ray error circle. According to Stephen et al. (2006) this is the lowerfrequency counterpart of the high-energy emission with a very high degree of probability. Given the arcsecond precision with which the position of soft X-ray sources is available, this technique reduces the sky area for the search for the optical counterpart by a factor $>10^{3}$. This easily allows us to pinpoint the actual optical counterpart on which we could, eventually, perform optical spectroscopy to determine its nature. Our survey detected 158 AGNs of a different type. Since the study of the stellar populations was not the principal aim of the investigation when the spectra were acquired, many of them are characterized by a short exposure time that does not guarantee either a quality or a signal-to-noise ratio $(\mathrm{S} / \mathrm{N})$ suitable for performing a reliable analysis of their stellar population properties. Out of the initial sample of optical counterparts of AGNs, we considered only the extracted spectra with $S / N \geq 20$ for which the important absorption lines (i.e. $\mathrm{H} \beta, \mathrm{Mg}$, and $\mathrm{Fe}$ ) were not strongly contaminated by broad-band emission lines or residual sky subtraction for the following analysis. Therefore, the final sample was comprised of 20 objects, classified mostly as Sy2, with soft and hard X-ray emission detected, and with gas emission lines measured. All these galaxies lie in the redshift range of $0.008 \leq z \leq 0.3$, and their properties are listed in Table 1.

\subsection{The data}

The spectroscopic observations of the sample galaxies were carried out with a variety of setups using different telescopes. These were as follow:

- the $3.58 \mathrm{~m}$ Telescopio Nazionale Galileo (TNG) in La Palma, Spain; 
- the $2.1 \mathrm{~m}$ telescope of the Observatorio Astronomico Nacional in San Pedro Martir, Mexico;

- the $1.5 \mathrm{~m}$ at the Cerro Tololo Interamerican Observatory (CTIO), Chile; and

- the $1.52 \mathrm{~m}$ Giandomenico Cassini telescope of the Astronomical Observatory of Bologna in Loiano, Italy.

Some spectra were also retrieved from the Sloan Digital Sky Survey (SDSS) archive (Adelman-McCarthy et al. 2006, 2008) and from the Six-degree Field Galaxy Survey (6dFGS) archive (Jones et al. 2004).

We refer to Papers I-XIII for detailed explanations on the observing setup, data reduction, calibration, and previous analysis. In Col. 6 of Table 1, the reference paper for each object is listed. In this paper, we just summarized the basic useful properties of the spectra used in the following analysis.

The sample consists of 20 spectra of galaxies acquired in long slit mode. The wavelength range between 3800 and $7500 \AA$ was covered with a reciprocal dispersion between $\sim 0.8$ and $\sim 5.7 \AA$ pixel $^{-1}$ after pixel binning. This corresponds to an instrumental velocity dispersion within the range $61 \mathrm{~km} \mathrm{~s}^{-1} \lesssim$ $\sigma_{\text {inst }} \lesssim 300 \mathrm{~km} \mathrm{~s}^{-1}$ at $5500 \AA$.

\section{Measurements of the stellar populations}

The stellar population properties, namely age and metallicity, were measured mainly from the following absorption features: $\mathrm{H} \alpha$ line $(\lambda 6563 \AA), \mathrm{H} \beta$ line $(\lambda 4861 \AA), \mathrm{H}_{\gamma}$ line $(\lambda 4340 \AA)$, $\mathrm{H}_{\delta}$ line $(\lambda 4102 \AA), \mathrm{Mg}$ I line triplet $(\lambda \lambda$ 5164, 5173, $5184 \AA)$, and Fe lines $(\lambda \lambda 5270,5335 \AA)$. With few exceptions, it was not possible to obtain information either from the blue part ( $\$ 3800 \AA$ ) of the spectrum because of the low efficiency of the optics, or from the red part ( $\gtrsim 7500 \AA$ ) due to the residuals of the strong emission lines of the sky in this region.

As done by Onodera et al. (2012), we applied the penalized pixel fitting (pPXF; Cappellari \& Emsellem 2004²), including the linear regularization of the weights (Press et al. 1992) and the Gas AND Absorption Line Fitting (GANDALF, Sarzi et al. 2006) IDL $^{3}$ packages which are adjusted for the sample spectra, to derive both the distribution of the mass fraction in different age and metallicity bins.

Even if the featureless continuum and broad lines are weak or absent in Sy2 galaxies (Lawrence et al. 1987), we allowed the code to use also broad band components in the fitting procedure. To account for the effect of dust and possible residuals of the data reduction procedure, we adopted a low order multiplicative polynomial in the template fitting. This has the advantage to make our method more sensitive to the absorption lines than to the continuum shape and, therefore, less sensible to the effects of reddening. However, we could not completely rule out the possibility of underestimating the weight of very reddened young stellar components in deriving the composite stellar populations.

For each spectrum, we fitted a linear combination of 156 template stellar spectra (Vazdekis et al. 2010) from the MILES (Sánchez-Blázquez et al. 2006) library ( $F W H M=2.54 \AA$ spectral resolution Beifiori et al. 2011) to the observed galaxy spectrum by performing a $\chi^{2}$ minimization in pixel space. Since the resolution of the sample spectra spans a wide range of values

\footnotetext{
2 Program available on http://www-astro.physics.ox.ac.uk/ $\sim \mathrm{mxc} / \mathrm{idl} /$

3 Interactive Data Language is distributed by Exelis Visual Information Solutions.
}

(always lower than the template stellar spectra) for each galaxy, it was necessary to convolve each template with the line-of-sight velocity distribution (LOSVD) and to rebin both the template and the galaxy spectrum to match their dispersions before running the fitting code.

We adopted the Salpeter initial mass function (Salpeter 1955), the 26 ages ranging from $1 \mathrm{Gyr}$ to $17 \mathrm{Gyr}$, and six metallicities $[\mathrm{M} / \mathrm{H}]$ from -1.71 to 0.22 . Simultaneously, we fitted the observed spectra using emission lines in addition to the stellar templates.

The extracted spectra on which we performed the measurement were the same used to derive the emission line properties in Papers I-XXIII. This guarantees the analysis to be in the same spatial regions used for the detection and measurements of the emission lines. The linear scale on which we were measuring galaxy properties depends on the combination of redshift and slit aperture and ranges from $200 \mathrm{pc}$ to $2 \mathrm{kpc}$. Swift J0811.5+0937 is the only exception, having a linear scale of $6 \mathrm{kpc}$. Based on the above, the region we were investigating was large enough to allow a study of the stellar populations of the bulge central region surrounding the AGN in almost all the cases.

The example of the fitting procedure shown in Fig. 2 (left panel) refers to galaxy PBC J0954.8+3724 and proves the good quality of the fit we obtained for all the galaxies. From the fitted single stellar population (SSP), we, then, derived the stellar mass fraction within each age and metallicity interval (right panel).

\section{Results and discussion}

We applied the procedure described in Sect. 3 to all the galaxies in our sample. In general, the distribution of the age and metallicity templates used by the code was, smooth for most of the galaxies and indicated that the bulk of stars in the considered region tended to be old.

To quantify this effect, we derived the mass-weighted age $\langle t / \mathrm{Gyr}\rangle_{M}$ and the mass-weighted metallicity $\langle[\mathrm{M} / \mathrm{H}]\rangle_{M}$ of its stellar population for each galaxy. These values are listed in Table 2 and plotted in Figs. 3-5. The color scale refers to the mass fraction in each bin of age and metallicity. The errors on the age and metallicity given in Table 2 were obtained from photon statistics and CCD readout noise, and they were calibrated through a series of Monte Carlo simulations.

In detail, almost all galaxies are characterized by an old stellar population with an age range from $8.4 \mathrm{Gyr}$ to 15.7 Gyr. There are only two galaxies (PBC J1546.5+6931 and $\mathrm{PBC} \mathrm{J} 0041.6+2534)$ with $\langle t / \mathrm{Gyr}\rangle_{M}<7$. However, PBC J1546.5+6931 shows a clear bimodality in the distribution and, for this reason, $\langle t / \mathrm{Gyr}\rangle_{M}$ and $\langle[\mathrm{M} / \mathrm{H}]\rangle_{M}$ are not properly describing its stellar population. Therefore, we independently derived the typical parameters of the two distinct stellar populations for this object. The younger one is characterized by $\langle t / \mathrm{Gyr}\rangle_{M}=3.6$ and $\langle[\mathrm{M} / \mathrm{H}]\rangle_{M}=0.18$, and it contributes to $\sim 70 \%$ of the total mass of the galaxy. The remaining $\sim 30 \%$ of the galaxy mass is due to a older population $\left(\langle t / \mathrm{Gyr}\rangle_{M}=14.8\right)$ with subsolar metallicity $\left(\langle[\mathrm{M} / \mathrm{H}]\rangle_{M}=-0.27\right)$.

Even though this is the most representative example of bimodality in the stellar population distribution, there are another two galaxies, namely PBC J0919.9+3712 and PBC J1345.4+4141, that show a second stellar population accounting for more than $10 \%$ of the total galaxy mass. The final results for the three galaxies with two separate stellar populations are listed in Table 3. As can noted in Table 3, the less massive component is a young one for both PBC J0919.9+3712 and PBC J1345.4+4141, which accounts for $12 \%$ and $15 \%$ of the 


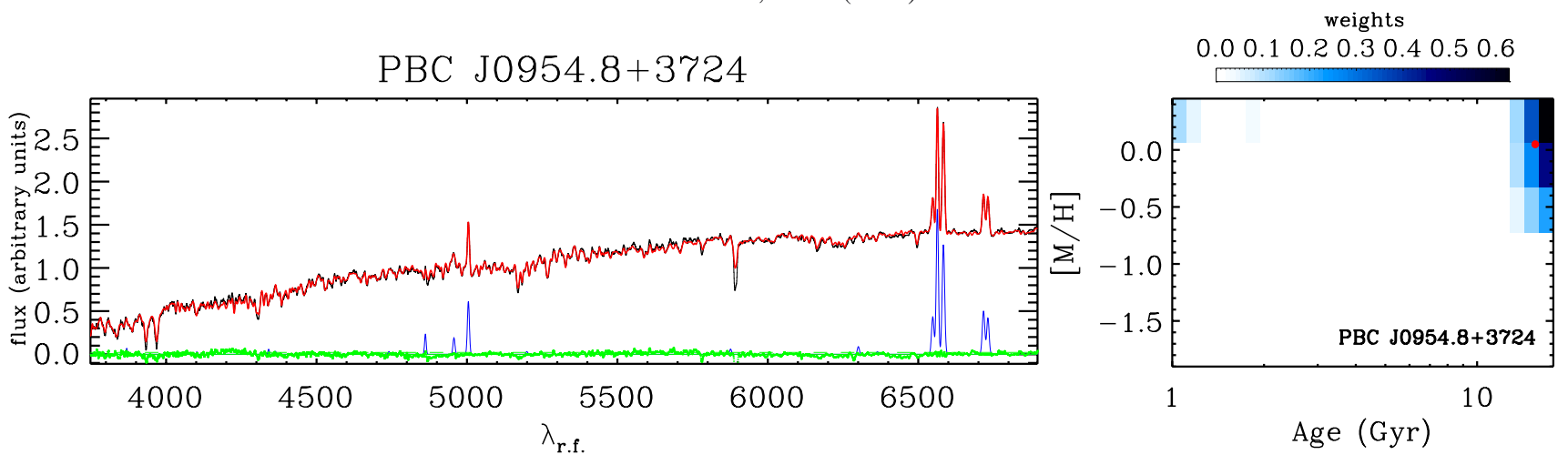

Fig. 2. Left panel: the galaxy spectrum (black line) is compared to the fitted linear combination of template stellar spectra (red line) and emission lines (blue line). The green dots show the residuals. Right panel: age and metallicity obtained from the spectral fitting. The color scale refers to the mass fraction in each bin of age and metallicity. The red dot represents the mass-weighted age $\langle t / \text { Gyr }\rangle_{M}$ and the mass-weighted metallicity $\langle[\mathrm{M} / \mathrm{H}]\rangle_{M}$.

Table 2. Mass-weighted age and metallicity measured for the galaxies in our sample.

\begin{tabular}{crr}
\hline $\begin{array}{c}\text { Galaxy } \\
\text { name }\end{array}$ & \multicolumn{1}{c}{$\begin{array}{c}\text { Age } \\
(\mathrm{Gyr})\end{array}$} & \multicolumn{1}{c}{$\begin{array}{c}\text { Metallicity } \\
{[\mathrm{M} / \mathrm{H}]}\end{array}$} \\
\hline IGR J01528-0326 & \multicolumn{1}{c}{$(2)$} & \multicolumn{1}{c}{$(3)$} \\
IGR J02524-0829 & $15.2 \pm 0.8$ & $0.13 \pm 0.04$ \\
IGR J04451-0445 & $9.6 \pm 2.1$ & $0.16 \pm 0.02$ \\
IGR J18244-5622 & $12.8 \pm 1.1$ & $-0.17 \pm 0.04$ \\
IGR J18308+0928 & $11.5 \pm 1.3$ & $0.21 \pm 0.02$ \\
PBC J0041.6+2534 & $5.0 \pm 1.1$ & $0.19 \pm 0.03$ \\
PBC J0759.9+2324 & $14.4 \pm 0.7$ & $0.15 \pm 0.01$ \\
PBC J0919.9+3712 & $14.5 \pm 0.6$ & $0.06 \pm 0.02$ \\
PBC J0954.8+3724 & $15.5 \pm 1.4$ & $0.04 \pm 0.02$ \\
PBC J1246.9+5432 & $10.0 \pm 0.9$ & $0.21 \pm 0.01$ \\
PBC J1335.8+0301 & $14.5 \pm 0.7$ & $0.20 \pm 0.02$ \\
PBC J1344.2+1934 & $15.6 \pm 1.0$ & $0.04 \pm 0.04$ \\
PBC J1345.4+4141 & $14.8 \pm 0.9$ & $0.05 \pm 0.03$ \\
PBC J1546.5+6931 & $6.8 \pm 1.7$ & $0.05 \pm 0.03$ \\
Swift J0134.1-3625 & $15.4 \pm 1.3$ & $0.11 \pm 0.03$ \\
Swift J0501.9-3239 & $15.2 \pm 1.4$ & $0.10 \pm 0.02$ \\
Swift J0601.9-8636 & $9.7 \pm 1.5$ & $0.00 \pm 0.04$ \\
Swift J0811.5+0937 & $14.6 \pm 1.1$ & $0.17 \pm 0.03$ \\
Swift J0911.2+4533 & $15.7 \pm 0.9$ & $-0.08 \pm 0.02$ \\
Swift J1238.9-2720 & $14.3 \pm 1.1$ & $0.06 \pm 0.02$ \\
\hline
\end{tabular}

Notes. Column (1): object name. Column (2): mass-weighted age in Giga-years derived from the stellar population fitting. Column (3): mass-weighted metallicity derived from the stellar population fitting.

total mass, respectively. The linear scales for these two objects are the smallest in our sample ( $200 \mathrm{pc})$, and this could have increased our sensibility in detecting a young component existing in the nuclear region of the galaxy.

It is interesting to note that an additional young component was also found by Cid Fernandes et al. (2004) in 30\% of the galaxies in their sample. Their percentage, which is higher than what we found, could be ascribed in that we were observing a larger region of the galaxy compared to what had been performed. For this reason, we were less sensitive to the young stellar populations that are possibly increasing towards the nucleus of the galaxy (Cid Fernandes et al. 2005). This could be the reason for the young component detected in PBC J0919.9+3712 and PBC J1345.4+4141. On the other hand, this is not the case for PBC J1546.5+6931, which shows a dominant young population and whose linear scale is bigger $(\sim 2 \mathrm{kpc})$. An explanation
Table 3. Mass-weighted age and metallicity measured for the young and old stellar populations considered separately.

\begin{tabular}{|c|c|c|c|c|c|}
\hline \multirow{3}{*}{$\begin{array}{c}\text { Galaxy } \\
\text { name }\end{array}$} & \multicolumn{2}{|c|}{ Age } & \multicolumn{2}{|c|}{ Metallicity } & \multirow{3}{*}{$\begin{array}{c}\text { Mass } \\
\text { old } \\
\%\end{array}$} \\
\hline & youn & old & young & old & \\
\hline & \multicolumn{2}{|c|}{ (Gyr) } & \multicolumn{2}{|c|}{$[\mathrm{M} / \mathrm{H}]$} & \\
\hline PBC J0919.9+3712 & 2.2 & 16.1 & 0.22 & 0.04 & 88 \\
\hline PBC J1345.4+4141 & 1.7 & 16.8 & 0.13 & 0.02 & 85 \\
\hline PBC J1546.5+6931 & 3.6 & 14.8 & 0.18 & -0.27 & 30 \\
\hline
\end{tabular}

Notes. Column (1): object name. Column (2): mass-weighted age of the young and old stellar component. Column (3): mass-weighted metallicity of the young and old stellar component. Column (4): percentage of the total mass ascribed to the old stellar population.

for the global young stellar population of this galaxy must be determined from its formation and evolution history.

Finally, the old ages that we derived for the majority of the galaxies in the sample agree with the results obtained for a large sample of infrared selected AGNs by Chen et al. (2009). Those objects, spanning different spectral classes and luminosities, all clearly show the old stellar population dominating the total mass and no relevant contributions from the young one. These results indirectly confirm that Sy2s reside very close to or even lie in the red sequence of galaxies (Schawinski et al. 2007).

The values of the mass-weighted age and metallicity that we obtained when considering only one stellar population (i.e. the one dominating the mass) are shown in Fig. 6 on the left and right panel, respectively. The large majority of the sample galaxies are characterized by a slightly supersolar massweighted metallicity (Fig. 6, right panel). The number distribution has a median value of $[\mathrm{M} / \mathrm{H}]=0.08$ and spreads from super $([\mathrm{M} / \mathrm{H}]=0.2)$ to subsolar values $([\mathrm{M} / \mathrm{H}]=-0.2)$. However, it should be noted that the fit used several different metallicities for the SSP, suggesting the possible existence of more metallicity components. Even when considering two distinct stellar populations for PBC J0919.9+3712, PBC J1345.4+4141, and PBC J1546.5+6931, the values of the metallicity remain inside this range (Table 3 ). Once again, this is consistent with the analysis done by Chen et al. (2009) and La Mura et al. (2012), who claimed increasing metallicity from starburst toward Seyferts and LINERs.

As a further step, we converted the mass-weighted ages and metallicities to the corresponding luminosity-weighted values. To perform the conversion, we adopted the $\mathrm{M} / \mathrm{L}$ ratios tabulated 


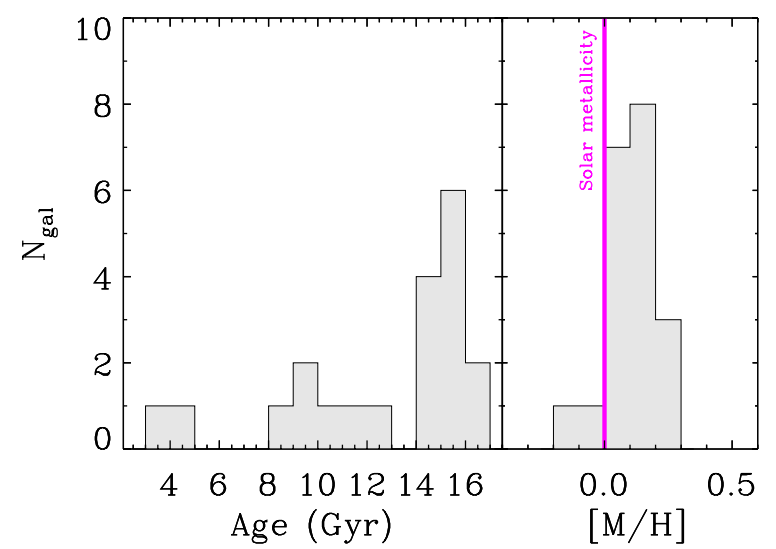

Fig. 6. Distribution of mass-weighted ages (left panel) and metallicities (right panel) for the dominant stellar component in the sample of galaxies. The magenta vertical line indicates the value of the solar metallicity.

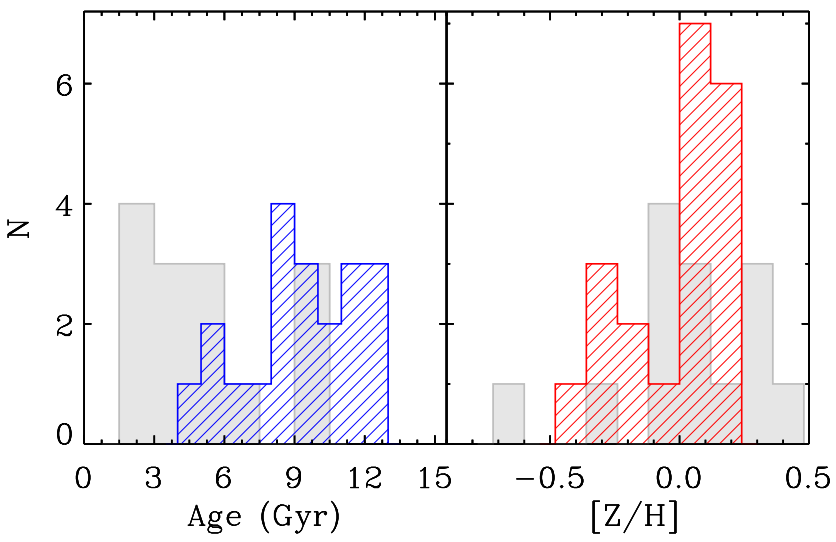

Fig. 7. Shaded histograms show the distribution of luminosity-weighted ages (left panel) and metallicities (right panel) for our sample of galaxies compared to the corresponding values derived by Morelli et al. (2008) for a similar sample of spirals (grey solid histogram).

for the SDSS $g$ filter by Maraston (2005). As we expected, the luminosity-weighted ages are slightly younger than the massweighted ones, but they remain, with few exceptions, globally old spanning the range between 6 and 12 Gyr. In Fig. 7, we plotted the histogram of the luminosity-weighted ages compared to those derived for the high surface brightness (HSB) sample in Morelli et al. (2008), which are characterized by a similar morphological type distribution.

Even though the number of galaxies in our sample does not allow us to trace a firm statistical conclusion, it is interesting to note that the bulges in our sample are globally older than those hosted in normal spirals. The metallicity of the bulges of LSB discs spans a large range of values from high $([\mathrm{Z} / \mathrm{H}]=$ $0.30 \mathrm{dex})$ to subsolar $([\mathrm{Z} / \mathrm{H}]=-0.2 \mathrm{dex})$ with a peak around slightly super-solar values (Fig. 7, right panel). The distribution of the metallicity in the galaxy sample is, instead, similar to the one derived for the bulges of HSB galaxies (Morelli et al. 2008).

We performed an additional analysis by looking for a possible correlation between the mass-weighted ages, metallicities, and the morphological type of our galaxies. Cid Fernandes et al. (2004) did not find any relevant correlation between the host morphology and the stellar population in the nuclear region of Sy2s, but the situation is less clear in the case of non-active galaxies. Studying a sample of spiral galaxies, Thomas \& Davies (2006) and Morelli et al. (2012) did not observe any correlation between the age and metallicity of the stellar population in

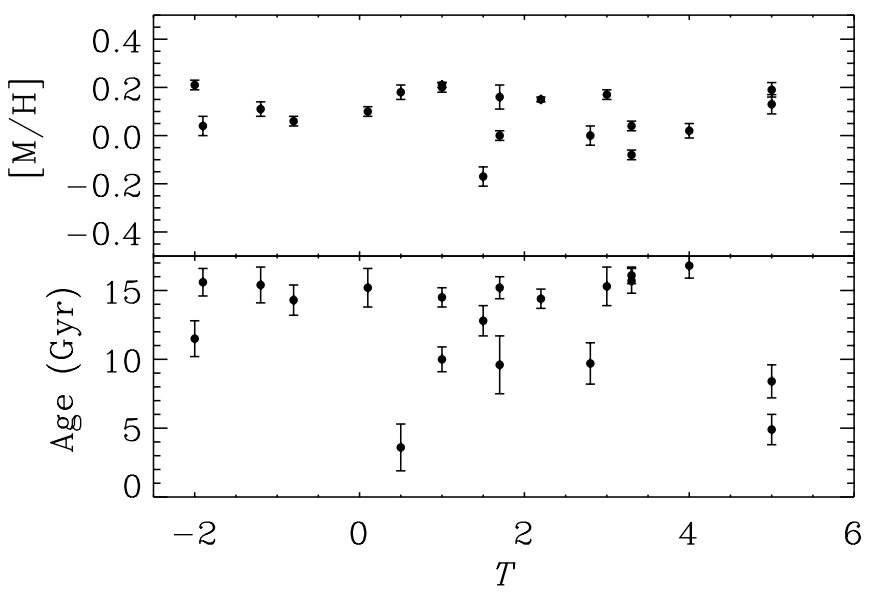

Fig. 8. Correlation between the morphological type and the stellar population properties. Top panel: the values for the mass-weighted metallicities are plotted as a function of the morphological type T. Bottom panel: same as in top panel for the mass-weighted ages in Gyr.

the central region of the bulge and galaxy morphology, whereas Ganda et al. (2007) and Morelli et al. (2008) found a mild correlation with the early-type galaxies $(T<0)$ being older and more metal rich than spirals $(T \geq 0)$. The galaxies in our sample span a wide range of $T$ type values $(-2 \leq T \leq 5)$ that are homogeneously distributed without any decreasing trend in number when going from the early to the late type, as observed in the Storchi-Bergmann et al. (2001) sample.

We did not observe any relevant trends between the galaxy morphological type and the age or metallicity of our galaxies (Fig. 8). In some way, it is tempting to say that the AGN feedback acts homogenizing the stellar populations in the central (few kpc) region of galaxies with different morphological type. However, the low number of galaxies with $T \leq 0$ and the shallow relation between morphology and stellar populations showed by non-active galaxies, prevented us from claiming any strong conclusion on this aspect.

\section{Summary and conclusions}

In the past years, our collaboration unveiled the nature of more than $250 \mathrm{X}$-ray emitting sources; 158 of which were identified as AGNs. In this paper, we presented the stellar population analysis of the host galaxy, which was performed on a carefully selected sample of 20 objects with the goal of understanding the still debated connection between the central AGN and the properties of the host galaxy, and the effect of the AGN feedback. The detailed analysis of the stellar population can give more important constraints on this topic.

The area on which we measured the galaxy properties ranges from $200 \mathrm{pc}$ to $2 \mathrm{kpc}$. This allowed us to measure the stellar populations of the bulge close to the center of the galaxy, where the effects of the AGN feedback on the host are expected to be relevant.

The spectral fitting method, based on PPXF and GANDALF, was applied to the galaxy spectra to measure the stellar populations properties of the sample. In particular, we obtained a massweighted age and a mass-weighted metallicity for each galaxy. The values of the mass-weighted metallicity span the range of $-0.2 \leq[\mathrm{M} / \mathrm{H}] \leq 0.2$ with a median value of $[\mathrm{M} / \mathrm{H}]=0.08$. The large majority of our objects (19 galaxies out of 20 , or $95 \%$ of the galaxies) show an old stellar population with ages older than 8 Gyr. Three of them are characterized by a bimodal distribution 
with a non-negligible contribution from young stars. We found that PBC J1546.5+6931 is dominated by a young stellar population which accounts for $\sim 70 \%$ of the total mass, while the young stellar component is less massive than the old one in the case of PBC J0919.9+3712 and PBC J1345.4+4141. For the former galaxy the nature of the young stellar component is probably related to the formation and evolution of the galaxy itself. Regarding the latter galaxies, there would be a possible starforming activity in their central region. Even after investigating the luminosity-weighted ages for the galaxies in our sample, the old nature of their stellar population is confirmed. The comparison of their ages with those obtained for a similar sample in terms of morphological type showed that bulges hosted in AGN are globally older than those hosted in non-active counterparts. However, it should be noted that, as expected, the contribution of the young component to the total light of the galaxy is greater than that to the total mass.

Our results suggest that AGN feedback acts on the first kpc of the galaxy, decreasing the efficiency of its star formation through different processes. The combination of truncation and suppression (Schawinski et al. 2009) could be responsible for disrupting the gas star-forming reservoir (Davis et al. 2012) in early times, when the gas is wiped out from the strong AGN emission in the center (Di Matteo et al. 2005; Springel et al. 2005; Fontanot et al. 2011). In recent times, the phase of the AGN regulates and quenches the residual star formation of the host galaxy (Sturm et al. 2011), maintaining the global red color and old stellar populations that are also observed in this work.

Radiative feedback and cooling flow of gas to the center could also, as proposed by Ciotti et al. (2010) and Novak et al. (2011), exhaust the gas in the few central kpcs region of the galaxy and decrease the star formation. The final consequence of this process is that the bulk of the observed stellar populations in this region is, with very few exceptions, old.

These scenarios are indirectly supported by the lack of relations between the stellar population properties and the morphological ones, in the sense that in the central region the existence of an AGN influences the properties of the galaxy much more than the formation and evolution, as described by the morphological type.

Acknowledgements. We thank Valentin Ivanov and Lodovico Coccato for useful discussions. L.M. acknowledges financial support from Padua University grant CPS0204. L.M. and V.C. acknowledge the Universidad Andrés Bello in Santiago del Chile for hospitality while this paper was in progress. P.P. and R.L. is supported by the INTEGRAL ASI-INAF grant No. 033/1070. N.M. acknowledges the Pontificia Universidad Católica de Chile for hospitality while this paper was in progress. D.M. and G.G. are supported by the Basal CATA Center for Astrophysics and Associated Technologies PFB-06. G.G. is supported by fondecyt grant 1120195 .

\section{References}

Adelman-McCarthy, J. K., Agüeros, M. A., Allam, S. S., et al. 2006, ApJS, 162, 38

Adelman-McCarthy, J. K., Agüeros, M. A., Allam, S. S., et al. 2008, ApJS, 175, 297

Barthelmy, S. D. 2004, in SPIE Conf. Ser. 5165, eds. K. A. Flanagan, \& O. H. W. Siegmund, 175

Beifiori, A., Maraston, C., Thomas, D., \& Johansson, J. 2011, A\&A, 531, A109 Bird, A. J., Bazzano, A., Bassani, L., et al. 2010, ApJS, 186, 1

Bluck, A. F. L., Conselice, C. J., Almaini, O., et al. 2011, MNRAS, 410, 1174

Cappellari, M., \& Emsellem, E. 2004, PASP, 116, 138

Chen, X. Y., Liang, Y. C., Hammer, F., Zhao, Y. H., \& Zhong, G. H. 2009, A\&A, 495,457
Cid Fernandes, R., Gu, Q., Melnick, J., et al. 2004, MNRAS, 355, 273

Cid Fernandes, R., González Delgado, R. M., Storchi-Bergmann, T., Martins, L. P., \& Schmitt, H. 2005, MNRAS, 356, 270

Ciotti, L., Ostriker, J. P., \& Proga, D. 2010, ApJ, 717, 708

Cracco, V., Ciroi, S., di Mille, F., et al. 2011, MNRAS, 418, 2630

Cusumano, G., La Parola, V., Segreto, A., et al. 2010, A\&A, 524, A64

Davies, R. I., Müller Sánchez, F., Genzel, R., et al. 2007, ApJ, 671, 1388

Davis, T. A., Krajnović, D., McDermid, R. M., et al. 2012, MNRAS, 426, 1574

de Vaucouleurs, G., de Vaucouleurs, A., Corwin, H. G., et al. 1991, Third Reference Catalogue of Bright Galaxies (Berlin: Springer) (RC3)

Di Matteo, T., Springel, V., \& Hernquist, L. 2005, Nature, 433, 604

Draper, A. R., \& Ballantyne, D. R. 2011, ApJ, 740, 57

Faber, S. M., Willmer, C. N. A., Wolf, C., et al. 2007, ApJ, 665, 265

Feruglio, C., Maiolino, R., Piconcelli, E., et al. 2010, A\&A, 518, L155

Fontanot, F., Pasquali, A., De Lucia, G., et al. 2011, MNRAS, 413, 957

Ganda, K., Peletier, R. F., McDermid, R. M., et al. 2007, MNRAS, 380, 506

Gehrels, N., Chincarini, G., Giommi, P., et al. 2004, ApJ, 611, 1005

González Delgado, R. M., Heckman, T., \& Leitherer, C. 2001, ApJ, 546, 845

Ho, L. C., Filippenko, A. V., \& Sargent, W. L. W. 2003, ApJ, 583, 159

Hopkins, P. F., \& Hernquist, L. 2006, ApJS, 166, 1

Ivanov, V. D., Rieke, G. H., Groppi, C. E., et al. 2000, ApJ, 545, 190

Joguet, B., Kunth, D., Melnick, J., Terlevich, R., \& Terlevich, E. 2001, A\&A, 380,19

Jones, D. H., Saunders, W., Colless, M., et al. 2004, MNRAS, 355, 747

La Mura, G., Di Mille, F., Ciroi, S., Popović, L. Č., \& Rafanelli, P. 2009, ApJ, 693, 1437

La Mura, G., Bindoni, D., Ciroi, S., et al. 2012, MNRAS, 426, 1893

Landi, R., Masetti, N., Morelli, L., et al. 2007, ApJ, 669, 109

Lawrence, A., Watson, M. G., Pounds, K. A., \& Elvis, M. 1987, Nature, 325, 694

Maiorano, E., Landi, R., Stephen, J. B., et al. 2011, MNRAS, 416, 531

Mannucci, F., Cresci, G., Maiolino, R., Marconi, A., \& Gnerucci, A. 2010, MNRAS, 408, 2115

Maraston, C. 2005, MNRAS, 362, 799

Masetti, N., Palazzi, E., Bassani, L., Malizia, A., \& Stephen, J. B. 2004, A\&A, 426, L41

Masetti, N., Bassani, L., Bazzano, A., et al. 2006a, A\&A, 455, 11

Masetti, N., Mason, E., Bassani, L., et al. 2006b, A\&A, 448, 547

Masetti, N., Morelli, L., Palazzi, E., et al. 2006c, A\&A, 459, 21

Masetti, N., Pretorius, M. L., Palazzi, E., et al. 2006d, A\&A, 449, 1139

Masetti, N., Mason, E., Morelli, L., et al. 2008, A\&A, 482, 113

Masetti, N., Parisi, P., Palazzi, E., et al. 2009, A\&A, 495, 121

Masetti, N., Parisi, P., Palazzi, E., et al. 2010, A\&A, 519, A96

Masetti, N., Parisi, P., Jiménez-Bailón, E., et al. 2012, A\&A, 538, A123

McKernan, B., Ford, K. E. S., \& Reynolds, C. S. 2010, MNRAS, 407, 2399

Morelli, L., Pompei, E., Pizzella, A., et al. 2008, MNRAS, 389, 341

Morelli, L., Corsini, E. M., Pizzella, A., et al. 2012, MNRAS, 423, 962

Novak, G. S., Ostriker, J. P., \& Ciotti, L. 2011, ApJ, 737, 26

Onodera, M., Renzini, A., Carollo, M., et al. 2012, ApJ, 755, 26

Parisi, P., Masetti, N., Jiménez-Bailón, E., et al. 2009, A\&A, 507, 1345

Parisi, P., Masetti, N., Jiménez-Bailón, E., et al. 2012, A\&A, 545, A101

Paturel, G., Petit, C., Prugniel, P., et al. 2003, A\&A, 412, 45

Press, W. H., Teukolsky, S. A., Vetterling, W. T., \& Flannery, B. P. 1992,

Numerical recipes in FORTRAN. The art of scientific computing, 2nd edn. (Cambridge: University Press)

Rigopoulou, D., Mainieri, V., Almaini, O., et al. 2009, MNRAS, 400, 1199

Salpeter, E. E. 1955, ApJ, 121, 161

Sánchez-Blázquez, P., Peletier, R. F., Jiménez-Vicente, J., et al. 2006, MNRAS, 371,703

Sarzi, M., Rix, H.-W., Shields, J. C., et al. 2005, ApJ, 628, 169

Sarzi, M., Falcón-Barroso, J., Davies, R. L., et al. 2006, MNRAS, 366, 1151

Schawinski, K., Thomas, D., Sarzi, M., et al. 2007, MNRAS, 382, 1415

Schawinski, K., Lintott, C. J., Thomas, D., et al. 2009, ApJ, 690, 1672

Springel, V., Di Matteo, T., \& Hernquist, L. 2005, MNRAS, 361, 776

Stephen, J. B., Bassani, L., Malizia, A., et al. 2006, A\&A, 445, 869

Storchi-Bergmann, T., González Delgado, R. M., Schmitt, H. R., Cid Fernandes,

R., \& Heckman, T. 2001, ApJ, 559, 147

Sturm, E., González-Alfonso, E., Veilleux, S., et al. 2011, ApJ, 733, L16

Thomas, D., \& Davies, R. L. 2006, MNRAS, 366, 510

Tremonti, C. A., Moustakas, J., \& Diamond-Stanic, A. M. 2007, ApJ, 663, L77

Ubertini, P., Lebrun, F., Di Cocco, G., et al. 2003, A\&A, 411, L131

Vaona, L., Ciroi, S., Di Mille, F., et al. 2012, MNRAS, 427, 1266

Vazdekis, A., Sánchez-Blázquez, P., Falcón-Barroso, J., et al. 2010, MNRAS, 404, 1639

Winkler, C., Courvoisier, T. J.-L., Di Cocco, G., et al. 2003, A\&A, 411, L1

Pages 7 to 9 are available in the electronic edition of the journal at http://www . aanda. org 
L. Morelli et al.: Stellar population properties for a sample of hard X-ray AGNs
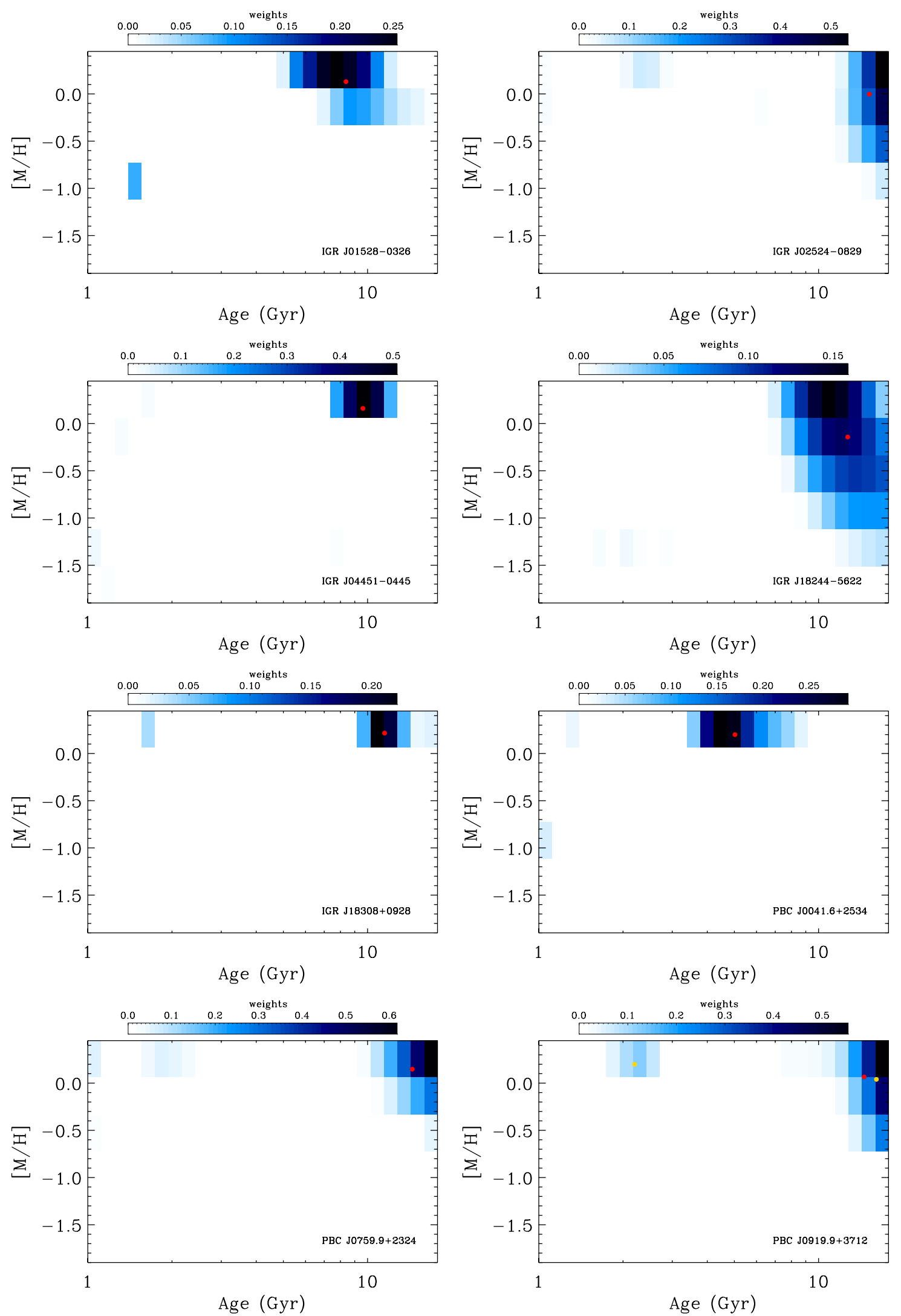

Fig. 3. Age and metallicity obtained from the spectral fitting for IGR J01528-0326, IGR J02524-0829, IGR J04451-0445, IGR J18244-5622, IGR J18308+0928, PBC J0041.6+2534, PBC J0759.9+2324, and PBC J0919.9+3712. The color scale refers to the mass fraction for each bin of age and metallicity. The red dot represents the mass-weighted age $\langle t / \mathrm{Gyr}\rangle_{M}$ and the mass-weighted metallicity $\langle[\mathrm{M} / \mathrm{H}]\rangle_{M}$ for each galaxy. The yellow dots represent the mass-weighted age $\langle t / \mathrm{Gyr}\rangle_{M}$ and the mass-weighted metallicity $\langle[\mathrm{M} / \mathrm{H}]\rangle_{M}$ in the case of two distinct stellar populations considered for the young and old component (see Sect. 4). 

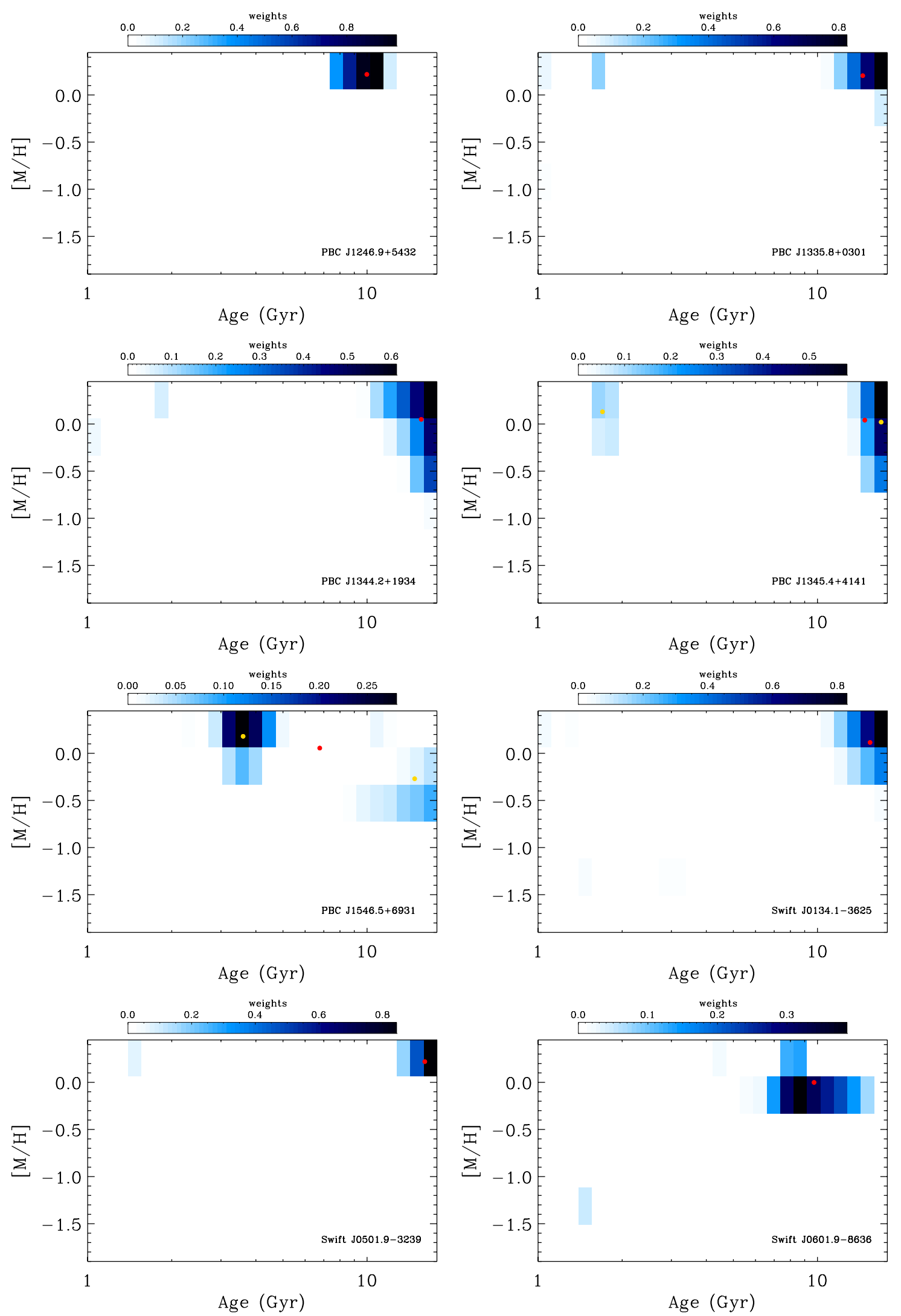

Fig. 4. Same as in Fig. 3 for PBC J1246.9+5432, PBC J1335.8+0301, PBC J1344.2+1934, PBC J1345.4+4141, PBC J1546.5+6931, Swift J0134.13625, Swift J0501.9-3239, and Swift J0601.9-8636. 
L. Morelli et al.: Stellar population properties for a sample of hard X-ray AGNs
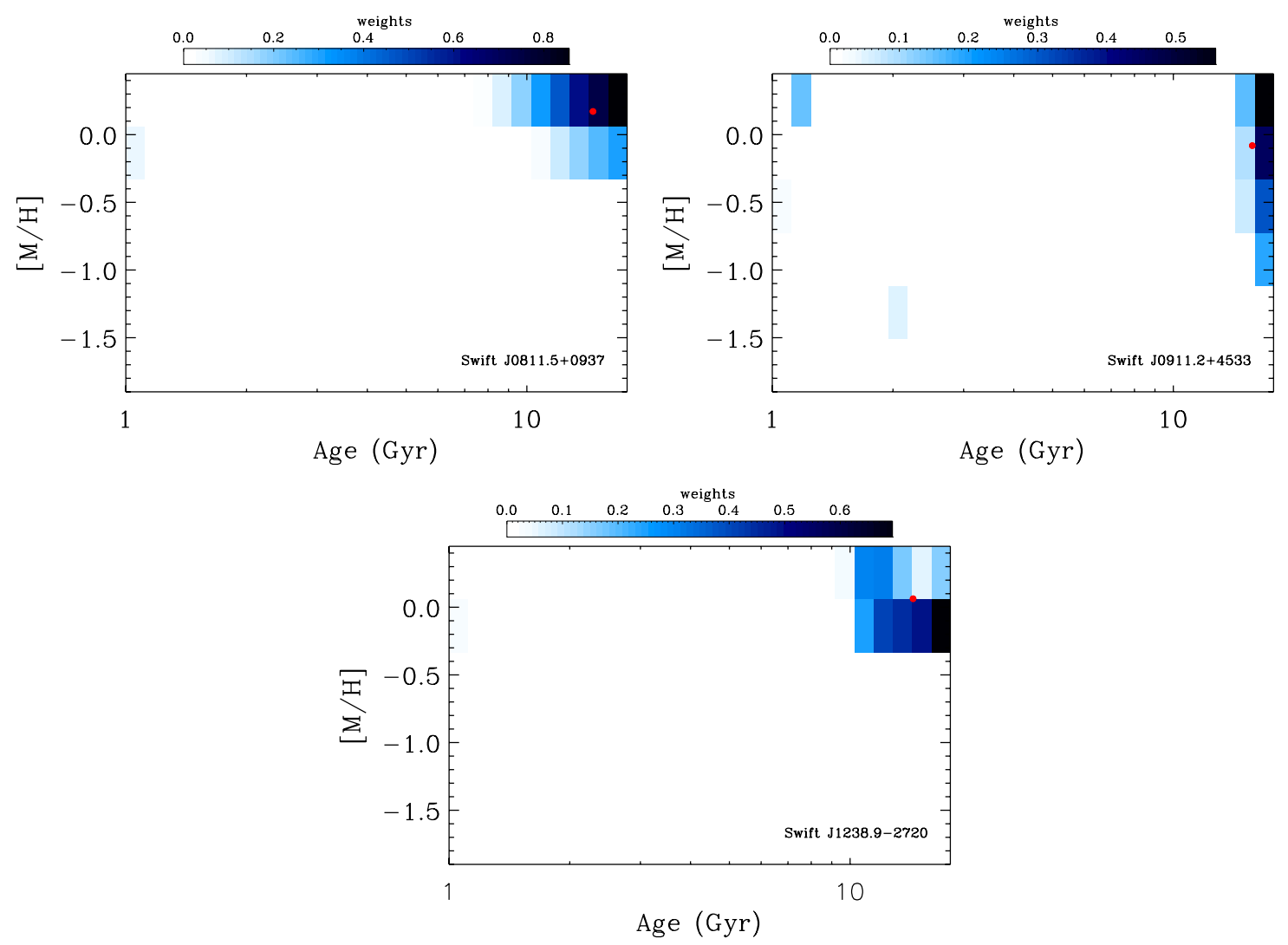

Fig. 5. Same as in Fig. 3 for Swift J0811.5+0937, Swift J0911.2+4533, and Swift J1238.9-2720.

Table 1. Properties of the galaxies in the sample.

\begin{tabular}{|c|c|c|c|c|c|c|c|}
\hline $\begin{array}{c}\text { Galaxy } \\
\text { name } \\
(1)\end{array}$ & $\begin{array}{c}\text { Galaxy } \\
\text { alt. name } \\
\text { (2) }\end{array}$ & Type & (4) & Class & (6) & $\begin{array}{c}B_{T} \\
(\mathrm{mag}) \\
(7)\end{array}$ & Source \\
\hline IGR J01528-0326 & PGC 6966 & $\mathrm{SA}(\mathrm{s}) \mathrm{c}$ ? & 5.0 & likely Sy2 & 0.017 & 14.11 & Masetti et al. (2008) \\
\hline IGR J02524-0829 & LEDA 10875 & $\mathrm{Sa}$ ? & 1.7 & Sy2 & 0.017 & 15.03 & Masetti et al. (2009) \\
\hline IGR J04451-0445* & LEDA 1053623 & $\mathrm{~S}$ ? & 1.7 & likely Sy2 & 0.076 & 17.10 & Masetti et al. (2010) \\
\hline IGR J18244-5622 & IC 4709 & $\mathrm{Sa}$ & 1.5 & Sy2 & 0.017 & 14.42 & Masetti et al. (2006c) \\
\hline IGR J18308+0928 & LEDA 1365707 & E? & -2.0 & Sy2 & 0.019 & 15.06 & Masetti et al. (2010) \\
\hline PBC J0041.6+2534 & NGC 0214 & $\mathrm{SAB}(\mathrm{r}) \mathrm{c}$ & 5.0 & Sy2/LINER & 0.015 & 12.94 & Parisi et al. (2012) \\
\hline PBC J0759.9+2324 & MCG+04-19-017 & $\mathrm{Sab}$ & 2.2 & Sy2 & 0.029 & 14.80 & Parisi et al. (2012) \\
\hline PBC J0919.9+3712 & IC 2461 & $\mathrm{Sb}$ & 3.3 & Sy2 & 0.008 & 14.63 & Parisi et al. (2012) \\
\hline PBC J0954.8+3724 & IC 2515 & $\mathrm{Sb}$ & 3.0 & Sy2 & 0.019 & 15.02 & Parisi et al. (2012) \\
\hline PBC J1246.9+5432 & LEDA 43101 & $\mathrm{Sa}$ & 1.0 & Sy2 & 0.017 & 13.60 & Parisi et al. (2012) \\
\hline PBC J1335.8+0301 & NGC 5231 & $\mathrm{SBa}$ & 1.0 & Sy2 & 0.022 & 14.29 & Parisi et al. (2012) \\
\hline PBC J1344.2+1934 & PGC 048674 & E? & -1.9 & Sy2/LINER & 0.027 & 15.58 & Parisi et al. (2012) \\
\hline PBC J1345.4+4141 & NGC 5290 & Sbc? & 4.0 & Sy1.9 & 0.009 & 13.30 & Parisi et al. (2012) \\
\hline PBC J1546.5+6931 & PGC 2730634 & $\mathrm{~S} ?$ & 0.5 & Sy1.9 & 0.037 & 16.08 & Parisi et al. (2012) \\
\hline Swift J0134.1-3625 & LEDA 5827 & SA0 & -1.2 & Sy2 & 0.029 & 14.03 & Parisi et al. (2009) \\
\hline Swift J0501.9-3239 & LEDA 17103 & SB0/a?(s) & 0.1 & Sy2 & 0.013 & 13.87 & Parisi et al. (2009) \\
\hline Swift J0601.9-8636 & LEDA 18394 & Sb? & 2.8 & Sy2 & 0.006 & 13.51 & Landi et al. (2007) \\
\hline Swift J0811.5+0937 & USNO-A2.0 0975-05763590 & - & - & XBONG & 0.286 & - & Parisi et al. (2009) \\
\hline Swift J0911.2+4533 & LEDA 2265450 & S? & 3.3 & Sy2 & 0.027 & 16.47 & Parisi et al. (2009) \\
\hline Swift J1238.9-2720 & ESO506G027 & S0 & -0.8 & Sy2 & 0.024 & 14.66 & Landi et al. (2007) \\
\hline
\end{tabular}

Notes. Column (1): name of the galaxy. Column (2): alternative name of the galaxy. Column (3): morphological type derived from the Third Reference Catalogue of Bright Galaxies (de Vaucouleurs et al. 1991) and from the Hyperleda database (Paturel et al. 2003). Column (4): numerical morphological type $T$ from the Hyperleda database. Column (5): classification of the AGN. Column (6): redshift of the object. Column (7): total apparent magnitude in the $B$-band derived from the Hyperleda database. Column (8): reference source for the galaxy. ${ }^{(*)}$ The identification of this object is still not confirmed. 